\title{
3-D IMAGE RECONSTRUCTION FROM EXPONENTIAL X-RAY PROJECTIONS USING NEUMANN SERIES
}

\author{
J.-M. Wagner, F. Noo \\ University of Liege, Belgium
}

\begin{abstract}
In two dimensions, the exponential X-ray transform has been wellstudied due to its applications of correcting attenuation effects in Single Photon Emission Computed Tomography (SPECT). Explicit inversion formulas have been known for over 15 years. The threedimensional (3D) case has not been as thoroughly examined, and inversion formulas are available for only a few of the wide range of possible 3D geometries. The Rotating Slant-Hole (RSH) SPECT geometry is a special case for which no inversion formula has yet appeared. This paper presents a general inversion formula for the 3D exponential X-ray transform using a Neumann series. The method applies to any geometry but convergence of the series depends on the exponential scalar and the size of the region-ofinterest. The derivation is presented in the context of the RSH SPECT geometry. Results from computer simulations are given.
\end{abstract}

\section{INTRODUCTION}

The reconstruction problem of a three dimensional (3-D) image from exponential X-ray (parallel-beam) projections is widely studied in 3-D Single Photon Emission Computed Tomography (SPECT) imaging systems. The main objective in SPECT imaging is to visualize the density distribution (also called emission map) of a radioactive tracer in some region of interest, like the heart or the brain. The emitted photons pass through different tissues (such as lungs, spine,...) and are attenuated before they interact with the detector. If the attenuation coefficient is constant in the emission region, the attenuated measured projections can be converted into exponential X-ray projections [1]. Thus, solving the reconstruction problem from exponential parallel projections provides a way to perform accurate attenuation correction and quantitative reconstruction of the emission map.

In conventional SPECT, parallel-hole collimators are used to collect attenuated projections. See figure 1 for a description of a parallel-hole collimator. The system collimator-detector is rotated around the z-axis of the region of interest (ROI) in order to sample the projections for directions located on a great circle. The measured projections are converted to exponential projections using multiplication factors derived from knowledge of the attenuation in the body. Then, the emission map is reconstructed using 2-D slice-by-slice reconstruction techniques which are now wellunderstood, especially thanks to the works of Tretiak and Metz [2], and Pan and Metz [3],[4].

Recently, the use of rotating slant-hole (RSH) collimators has been investigated [5]. The main interest of this configuration is to increase photon sensitivity and thus signal-to-noise ratio over standard parallel-hole collimator systems. See figure 2 for a description of a bilateral slant-hole collimator. Roughly, twice as many

\author{
R. Clackdoyle
}

University of Utah, USA

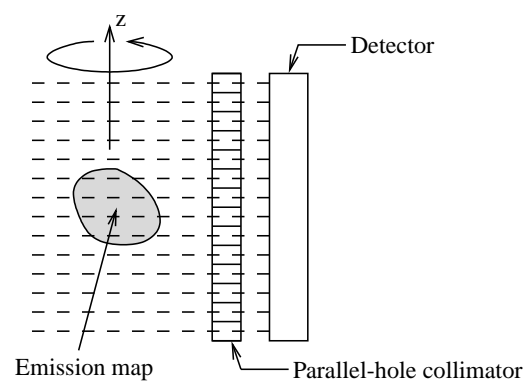

Fig. 1. Parallel-hole Collimator. The dotted lines indicate the directions of photons that pass through the collimator to the detector.

photons can be detected during the same acquisition period since the bilateral slant-hole collimator allows simultaneous collection of two projections. The system (slant-hole collimator)-detector is successively located at several fixed positions around the z-axis of the ROI. For each fixed position of the detector, the collimator is finely rotated about its axis as shown in figure 2. The combination of these two rotations defines the RSH SPECT acquisition geometry as described in [5]. The RSH SPECT geometry is a fully 3-D geometry which needs true 3 -D reconstruction techniques.

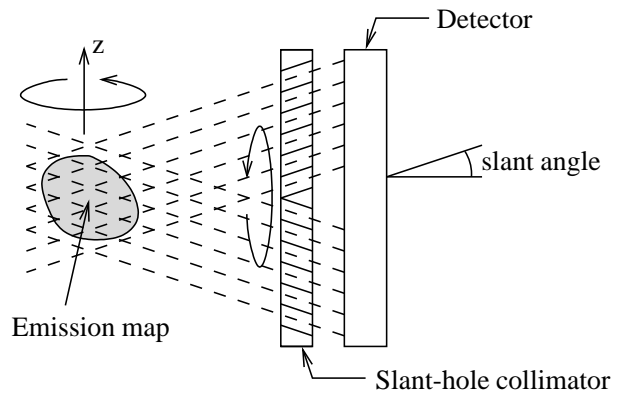

Fig. 2. A bilateral Slant-hole collimator. When the collimator is fixed, two parallel projections can be measured during the same acquisition period.

To our knowledge, only a few works concerning exact 3-D reconstruction from exponential X-ray projections have been published. Furthermore, these works only concern specific geometries. In [6] and [7], it is assumed that the projections are finely sampled on the full unit sphere. The algorithm described in [8] only deals with sets of projections containing great circles of the unit sphere. For more sophisticated geometry such as RSH SPECT, 
no exact reconstruction algorithm has been established so far.

In this paper, we present an exact inversion formula for the exponential X-ray transform in the particular case of the RSH geometry. The derivation of our algorithm is based on the knowledge of a filtered backprojection (FBP) algorithm for the non-attenuated case. The exponential parallel projections are first treated as nonattenuated projections by this FBP algorithm to give a first image $\widehat{f_{0}}$. Using some properties of the projection and backprojection operators, we have derived an exact inversion formula for the attenuated case in the form of a Neumann series with $\widehat{f_{0}}$ as the first term of the series.

This paper contains four sections. Section 2 gives a detailed description of the RSH SPECT geometry and defines the projection and backprojection operators. Section 3 describes our algorithm and provides mathematical details of its derivation. Simulations and reconstruction results are shown with a short discussion in section 4 .

\section{THE RSH SPECT GEOMETRY}

Figure 3 gives a description of the RSH SPECT geometry. In this figure, $\mathrm{O}$ is the origin of the image space and $z$ is assumed to be the main rotation axis of the geometry. $\underline{\gamma}_{d}$ is a unit vector in the $x y$ plane, attached to the detector and normal to its surface. The angle $\lambda_{i}$ specifies its position around the $\mathrm{z}$-axis. We will assume $N$ different positions $(i=1,2, \ldots, N)$ of the detector. Two unit orthogonal vectors $\underline{e}_{u}$ and $\underline{e}_{v}$ are parallel to the detector plane and attached to the slant-hole collimator. The angle $\varphi$ specifies the angular position of the collimator around the axis $\underline{\gamma}_{d}$ of the detector.

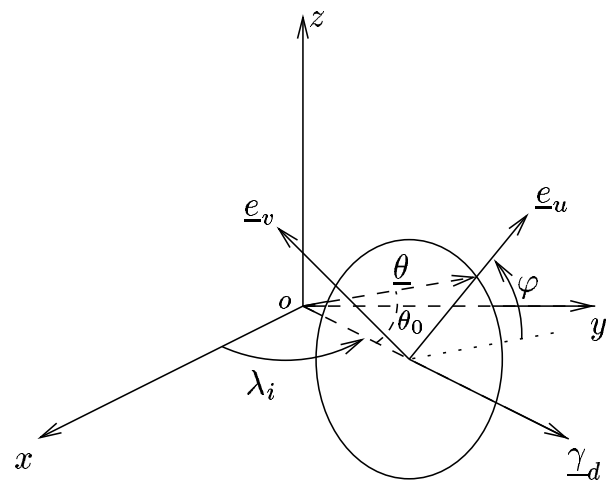

Fig. 3. Description of the RSH geometry.

The unit vector $\underline{\theta}$ defines the direction of the photons through the collimator to the detector. It is attached to the collimator and can be expressed in the form

$$
\underline{\theta}=\cos \theta_{0} \underline{\gamma}_{d}+\sin \theta_{0} \underline{e}_{u}
$$

where the constant $\theta_{0}$ is the slant angle of the collimator. The $\mathrm{RSH}$ geometry is mathematically defined by the trajectory $\Omega$ that $\underline{\theta}$ describes on the unit sphere when $\varphi$ is continuously varying from 0 to $2 \pi$ and for all values of $i$. This trajectory is composed of $N$ distinct circles of angular aperture $\theta_{0}$. In the following, the trajectory $\Omega$ is assumed to be complete, i.e. it intersects all great circles of the unit sphere [9].

\subsection{The projection operator}

We will let $f(\underline{x})$ denote the 3-D emission map to be imaged. The exponential projection operator $R_{\mu}$ is defined by

$$
\left[R_{\mu} f\right]\left(\lambda_{i}, \varphi, u, v\right)=\int_{-\infty}^{+\infty} d t f\left(u \underline{e}_{u}+v \underline{e}_{v}+t \underline{\theta}\right) e^{\mu t}
$$

where $\mu$ is the constant attenuation factor. The point $(u, v)=$ $(0,0)$ corresponds to the projection of the origin of the image space onto the detector plane in the direction $\underline{\theta}$. When $\lambda_{i}$ and $\varphi$ are fixed, $g_{\mu}\left(\lambda_{i}, \varphi, u, v\right)=\left[R_{\mu} f\right]\left(\lambda_{i}, \varphi, u, v\right)$ is a 2-D function of $u$ and $v$ known as an exponential X-ray projection of $f$. For $i=1, \ldots, N$ and $\varphi \in\left[0,2 \pi\left[, g_{\mu}\left(\lambda_{i}, \varphi, u, v\right)\right.\right.$ is a set of exponential projections and constitutes the data of the reconstruction problem.

\subsection{The backprojection operator}

The operator $R_{\mu}^{\#}$ is defined by

$$
\left[R_{\mu}^{\#} g_{\mu}\right](\underline{x})=\frac{1}{\cos \theta_{0}} \sum_{i=1}^{N} \int_{0}^{2 \pi} d \varphi g_{\mu}\left(\lambda_{i}, \varphi, \hat{u}, \hat{v}\right) e^{\mu \hat{t}}
$$

where

$$
\begin{aligned}
\hat{u} & =\underline{x} \cdot \underline{e}_{u}-\tan \theta_{0} \underline{x} \cdot \underline{\gamma}_{d} \\
\hat{v} & =\underline{x} \cdot \underline{e}_{v}, \\
\hat{t} & =\sec \theta_{0} \underline{x} \cdot \underline{\gamma}_{d}
\end{aligned}
$$

Geometrically, for each parallel projection, the point $(u, v)=$ $(\hat{u}, \hat{v})$ corresponds to the projection of $\underline{x}$ onto the plane of the detector in the direction $\underline{\theta}$. It is easily established that the operator $R_{\mu}^{\#}$ is the adjoint of the projection operator $R_{\mu}$. The backprojection operator is $R_{-\mu}^{\#}$ and $\left[R_{-\mu}^{\#} g_{\mu}\right](\underline{x})$ is the sum of the contributions of each projection for the point $\underline{x}$. Neither $R_{-\mu}^{\#}$ nor $R_{\mu}^{\#}$ is the inverse of the projection operator $R_{\mu}$.

\section{INVERSION FORMULA}

Our algorithm only holds if the set $\Omega$ of parallel exponential projections satisfies Orlov's condition, i.e. it intersects all great circles of the unit sphere [9]. In that case, an exact FBP algorithm exists for the non-attenuated case [11], [10]. It can be written in a functional form as follows

$$
f=R_{0}^{\#}\left(h_{0} \star_{2} g_{0}\right)
$$

where $\star_{2}$ is a 2-D convolution and $h_{0}\left(\lambda_{i}, \varphi, u, v\right)$ is the 2-D filter to apply to the non-attenuated $(\mu=0)$ projections $g_{0}\left(\lambda_{i}, \varphi, u, v\right)$. The analytical expression of $h_{0}$ can be found in [10]. For the exponential case $(\mu \neq 0)$, no reconstruction algorithm has been published so far.

\subsection{Algorithm description}

The exact inversion formula we propose for the attenuated case is in the form of a Neumann series

$$
f=(1+K+K K+K K K+\ldots) \widehat{f_{0}}
$$


where the first term of the series $\widehat{f}_{0}$ is obtained from $g_{\mu}$ by

$$
\widehat{f}_{0}(\underline{x})=\chi(\underline{x})\left[R_{-\mu}^{\#}\left(h_{0} \star_{2} g_{\mu}\right)\right](\underline{x})
$$

i.e. the exponential parallel projections are first filtered by $h_{0}$ and then backprojected using the $R_{-\mu}^{\#}$ operator. $\widehat{f_{0}}$ is obtained by multiplying the result of the backprojection by the support function $\chi$. $\chi(\underline{x})$ is a 3 -D function equal to one on the region of interest and zero outside.

$K$ is a linear operator defined by

$$
[K f](\underline{x})=\chi(\underline{x})\left(f \star_{3} w\right)(\underline{x})
$$

where $w(\underline{x})$ is defined by

$$
w(\underline{x})=\frac{1}{\cos \theta_{0}} \sum_{i=1}^{N} \int_{0}^{2 \pi} d \varphi h_{0}\left(\lambda_{i}, \varphi, \hat{u}, \hat{v}\right)\left(1-e^{-\mu \hat{t}}\right)
$$

with $\hat{u}, \hat{v}$ and $\hat{t}$ given by equations (4), (5) and (6) respectively. The inversion formula holds if the series is convergent. A sufficient condition for convergence is

$$
\|K\|=\sup _{f} \frac{\|K f\|}{\|f\|}<1
$$

where $\|K f\|$ is the norm of the function $K f$. It can be shown that this condition is met for small values of $\mu D$ where $D$ is the diameter of the region-of-interest. Given a $\mu$ value, exact reconstruction is thus possible when the radioactive tracer is confined to a sufficiently small region-of-interest.

\subsection{Algorithm derivation}

To derive our inversion formula, note that the projection and backprojection operators satisfy the property

$$
\left(R_{-\mu}^{\#} h\right) \star_{3} f=R_{-\mu}^{\#}\left(h \star_{2} g_{\mu}\right)
$$

for any filter $h\left(\lambda_{i}, \varphi, u, v\right)$ and any value of $\mu$ [6]. From equation (7), we obtain $\left[R_{0}^{\#} h_{0}\right](\underline{x})=\delta_{3}(\underline{x})$ where $\delta_{3}$ is the 3-D delta Dirac function.

In equation (13), we replace $h$ by the filter used in the nonattenuated case, i.e. $h_{0}$. We define the left hand side as $f_{0}$, and obtain

$$
\begin{aligned}
f_{0} & =R_{-\mu}^{\#}\left(h_{0} \star_{2} g_{\mu}\right) \\
& =\left(R_{-\mu}^{\#} h_{0}-\delta_{3}\right) \star_{3} f+f \\
& =-w \star_{3} f+f
\end{aligned}
$$

where we have replaced $\delta_{3}$ by $R_{0}^{\#} h_{0}$ and $w$ is a 3-D function defined by (11). We introduce the support function $\chi$ to be sure that $\chi f_{0}$ and $\chi\left(w \star_{3} f\right)$ are equal to zero outside the reconstruction grid when the inversion formula will be discretised. We write

$$
f=\widehat{f_{0}}+K f
$$

where we have used $\chi f \equiv f$ and the definitions (9), (10) of $\widehat{f_{0}}$ and $K$. Using this last relation, we can also write

$$
\begin{aligned}
f & =\widehat{f_{0}}+K\left(\widehat{f_{0}}+K f\right)=\widehat{f_{0}}+K\left(\widehat{f_{0}}+K\left(\widehat{f_{0}}+K f\right)\right) \\
& =\widehat{f_{0}}+K \widehat{f_{0}}+K K \widehat{f_{0}}+K K K \widehat{f_{0}}+\ldots
\end{aligned}
$$

which is exactly equal to (8).

\section{RESULTS AND DISCUSSION}

Figure 4 shows one slice of the simulated attenuation and emission maps we used to make simulations. On the attenuation map (upper-left figure), one sees the two lungs and the spine which have different attenuation coefficients. The upper-right figure shows the phantom modeling the heart. This phantom is the simulated emission map and consists of three ellipsoids, two of which model the ventricules with $20 \%$ of activity. Attenuation and emission maps are both represented on the lower-left figure. On the lower-right figure, the heart phantom is discretised on a grid of $60^{3}$ cubic voxels of side $2.5 \mathrm{~mm}$. It has been used as a reference for our simulations.
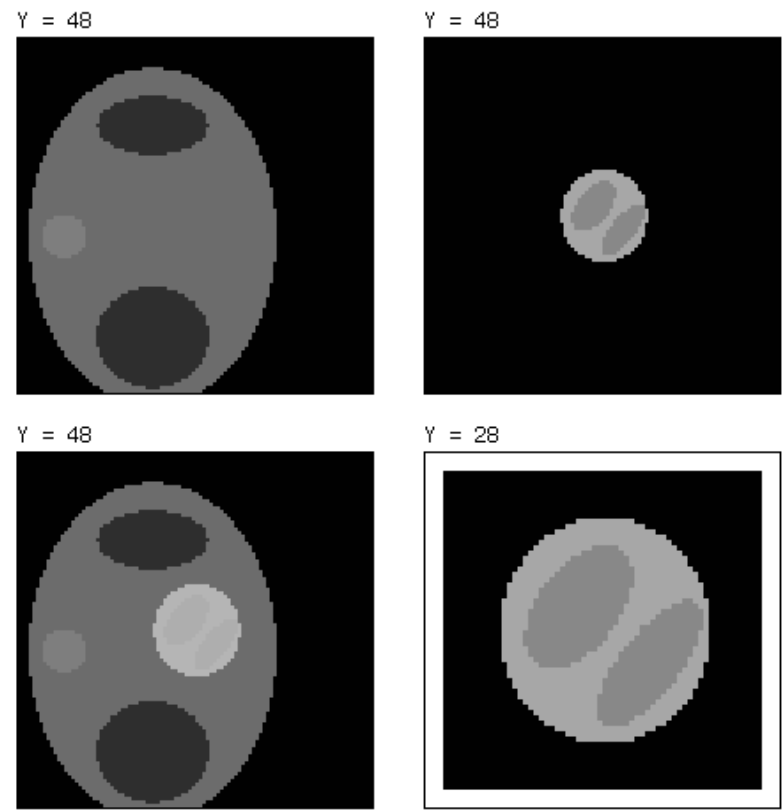

Fig. 4. Description of the simulated phantoms.

Attenuated parallel projections were simulated for the RSH SPECT geometry. We used 6 positions of the detector $\left(\lambda_{i}=i 60^{\circ}\right.$, $i=1, \ldots, 6)$. For each detector position, 32 positions of the collimator were simulated over 360 degrees with a uniform step of 11.25 degrees. The 192 projections were sampled on grids of $128^{2}$ pixels of side $1.5 \mathrm{~mm}$. The knowledge of the attenuation distribution allowed us to convert the attenuated projections into exponential projections with a constant attenuation factor $\mu$ equal to $0.0075 \mathrm{~mm}^{-1}$. In practice, most SPECT scanners can provide an accurate attenuation map.

The calculation of $w(\underline{x})$ with equation (11) was implemented by sampling the filter $h_{0}$ in the same way as the parallel projections were, i.e. we discretised $h_{0}$ in 1922 -D spatial filters. The backprojection routines used to calculate $\widehat{f}_{0}$ was also used to calculate $w$ thanks to $w(\underline{x})=\left[R_{0}^{\#} h_{0}\right](\underline{x})-\left[R_{-\mu}^{\#} h_{0}\right](\underline{x})$.

Figure 5 shows reconstructions achieved on grids of $60^{3}$ voxels of side $2.5 \mathrm{~mm}$. The upper-left figure shows the original heart phantom. The upper-right figure shows $\widehat{f}_{0}$. One can see that $\widehat{f}_{0}$ is not an exact reconstruction. The two lower figures shows reconstructions using the Neumann series with 2 and 3 terms, respectively. One can see improvements: the image support tends 
to be better defined and the gray levels tends to be more uniform in regions of constant activity. These preliminary results are encouraging. Further work concerning convergence conditions, discretization effects and stability in the presence of data noise is now required.
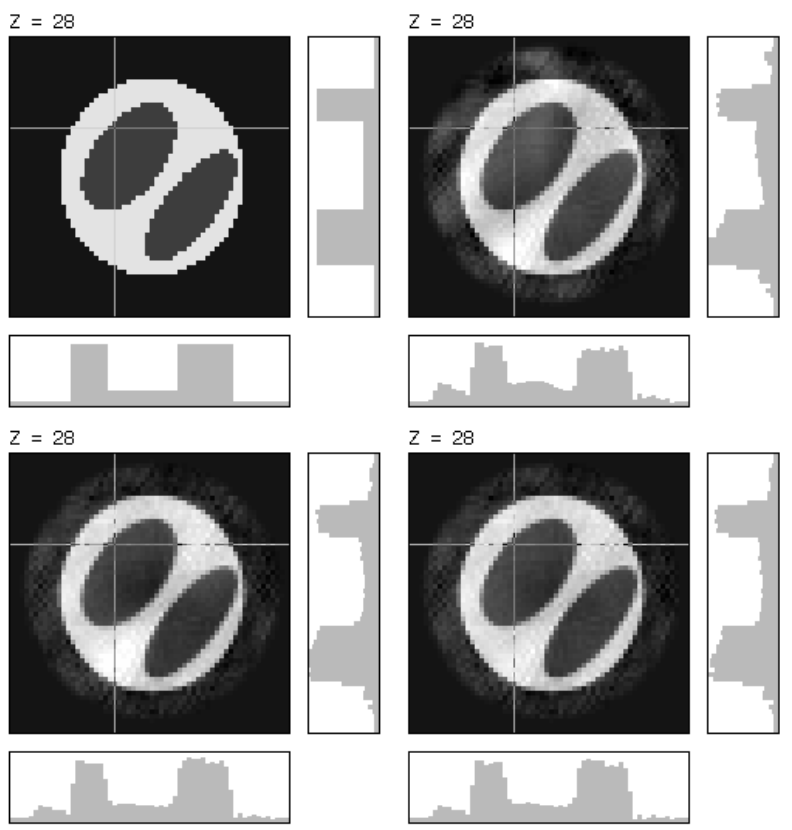

Fig. 5. Reconstruction of the heart phantom. Upper-left : ideal phantom. Upper-right : $\widehat{f_{0}}$. Lower-left : $\widehat{f}_{0}+K \widehat{f_{0}}$. Lower-right : $\widehat{f_{0}}+K \widehat{f_{0}}+K K \widehat{f_{0}}$.

\section{ACKNOWLEDGMENTS}

This work was partially supported by the National Institutes of Health, grant number R01 HL 55610. The work of F. Noo was supported by the Belgian National Fund for Scientific Research.

\section{REFERENCES}

[1] A. Markoe, "Fourier inversion of the attenuated X-ray transform”, SIAM J. Math. Anal., Vol. 15(4), 718-722, 1984.

[2] O. Tretiak, C. Metz, "The exponential Radon transform", SIAM, J. Appl. Math., Vol. 39(2), 341-354, 1980.

[3] C.E. Metz, X. Pan, "A unified analysis of exact methods of inverting the 2D exponential Radon transform, with implications for Noise control in SPECT", IEEE Trans. Med. Imag., vol. 14(4), december 1995.

[4] X. Pan, C.E. Metz, "Analysis of noise properties of a class of exact methods of inverting the 2D exponential Radon transform”, IEEE Trans. Med. Imag., vol. 14(4), december 1995.

[5] R. Clack, P.E. Christian, M. Defrise, A.E. Welch, "Image reconstruction for a novel SPECT system with rotating slanthole collimators". In Conf. Rec. 1995 Med. Imag. Conf., 1948-1952, 1996.
[6] I.A. Hazou, D.C. Solmon, "Inversion of the exponential Xray transform. I:Analysis", Math. Methods in the Applied Sciences, Vol. 10(10), 561-574 (1988).

[7] Y. Weng, G.L. Zeng, G.T. Gullberg, "Filtered backprojection algorithm for attenuated parallel and cone-beam projections sampled on a sphere", in Three-dimensional Image Reconstruction In Radiation and Nuclear Medecine, ed. P. Grangeat and J.-L. Amans (Dordrecht: Kluwer), 19-34, 1996.

[8] J.-M. Wagner, F. Noo, "TTR algorithm for the inversion of the exponential X-ray transform”. In Conf. Rec. 2000 Med. Imag. Conf., to be published.

[9] S.S. Orlov, "Theory of three dimensional reconstruction. 1. Conditions of a complete set of projections.", Sov. Phys. Crystallogr., Vol. 20, 312-314, 1975.

[10] Y.C. Wang, D.E. Wessel, E.C. Frey, B.M.W. Tsui, “A 3D Filtered Back-Projection Algorithm for SPECT using a MultiSegment Rotating Slant-Hole Collimator". In Conf. Rec. 2000 Med. Imag. Conf., to be published.

[11] M. Defrise, D.W. Townsend, R. Clack, "Three-dimensional image reconstruction from complete projections", Phys. Med. Biol. 34(5), 573-587, 1989. 